\title{
Techniques to Develop Genetically Improved Potato with Increased Resistance to Potato Late Blight (Phythophthora Infestans) A Review
}

\author{
Geletaw Kebede \\ College of Agriculture, Department of horticulture, Wolaita Sodo University, \\ P.O.BOX : 138, Wolaita Sodo, Ethiopia
}

\begin{abstract}
Infestation of potatoes with phythophthora infestans ,also known as "potato late blight "' is the most devastating potato disease world wide. Its occurrence often results in huge economic losses for potato producers. Current control measures involving extensive use of fungicides come with environmental costs. Efforts have been made to develop commercial potato varieties with increased resistance to p.infesting (the causal agent of late blight )using a variety of approaches .Due to the remarkable ability of p.infesting to over come resistance ,potato breeders have not yet succeeded in developing commercial potato varieties with resistance that is lasting. One approach where genetic engineering is used to 'stack' genes with broad spectrum resistance to p.infesting in commercial potato varieties, has been recently been employed as a means to create genetically improved potato varieties with more durable resistance.
\end{abstract}

DOI: $10.7176 / \mathrm{JNSR} / 12-18-01$

Publication date:September $30^{\text {th }} 2021$

\section{INTRODUCTION}

Potato (Solanum tuberosum L.) is one of the most important food crops in the world (Struik and Wiersema, 1999). In volume of production (347 million metric tons annually), it ranks fourth in the world after maize, rice and wheat, with an estimated production area of 18.9 million hectares (FAO, 2004). Among root crops, potato ranks first in volume produced and consumed, followed by cassava, sweet potato, and yam (FAO, 2004).

In terms of dry matter production per hectare (ha), potatoes are among the most productive crops grown in the developing countries. The tuber is known to supply carbohydrate, high quality protein, and a substantial amount of essential vitamins, minerals, and trace elements (Horton and Sawyer, 1985). In addition, the potato crop provides more nutritious food per unit land area, in less time, and often under more adverse conditions than other food crops. It is said to be one of the most efficient crops in converting natural resources, labour and capital into a high quality food with wide consumer acceptance (Horton, 1987). With its high potential to supply a cheap high quality food within a relatively short period of time, potato has the potential to improve appreciably the quality of the basic diet in rural and urban areas of

Ethiopia provided that a coordinated national effort is made to promote its production. The cultivated potato belongs to the family Solanaceae. It is originated in the high Andes of South America and was first cultivated in the vicinity of Lake Titicaca near the present border of Peru and Bolivia (Horton, 1987). It is believed to be introduced to Ethiopia around 1858 by a German botanist, Shimper (Pankhrust, 1964). Since then, it has become an important garden crop and currently, it is the second most important tuber crop grown in the country next to "Enset" (Ensete ventircosum L.) in terms of area coverage (Solomon, 1985). However, the current total area under potato production is estimated at 36,736 ha with an annual production of 385,258 metric tons and the national average yield is 10.5 tons/ha, which is very low compared to the world average of 16.4 tons/ha (FAO, 2004). Low soil fertility is one of the most important among the multitude of problems contributing to low yield of potato in Ethiopia

Many diverse and complex biotic,and abiotic,afactors have contributed to the existing of low productivity such as poor growing practices, lack of good quality planting materials, low use of inputs, poor control of disease and pests improper time of plantinand harvesting, rigid traditional food habit of the people and poor soil management practices can be mentioned as major constraints (Berga et al., )

The main objective of this litraeture are:-

To an overview on methods of increase resistance of potato to diseases by conventional as well as genetic engineering so as to increase production and decrease cost of production

\section{Phytophthora Infestans - 'late blight' - is the largest threat to potato cultivation}

Potato cultivation suffers from various diseases and pests, but 'late blight' forms the biggest threat. Other threats are nematodes (Globodera pallida, Globodera rostochiensis and Meloidogyne spp), and some bacterial diseases such as brown rot (Ralstonia solanacearum), ring rot (Clavibacter michiganensis) and blackleg (Dyckeya spp). Late blight is caused by the oomycete or water mould Phytophthora infestans, which is a fungus-like organism. 
Late blight is best known for the Great Famine it caused in Ireland around 1845. In a number of years the disease caused big potato crop failures in Ireland and proved to be a turning point in Irish history. About a million Irishmen died as a result of the famine and about the same number migrated, mostly to the U.S. to start a new life there. Phytophthora infestans emerged in Central America and crossed the Atlantic Ocean to Europe from there in 1843. The Great Famine took place only two years after.

The susceptibility of potatoes for Phytophthora infestans differs from variety to variety, but only very few varieties are characterized by complete resistance.

\subsection{Phytophthora infestans affects both plant and tuber, and is highly contagious}

Phytophthora infestans affects leaves, stems and tubers. When a plant is affected, lesions (spots on the leaves) occur on the leaves. How these spots look, depend on weather conditions. brown spots are lined by a yellow ring. In damp weather conditions, the underside of the leaf will show a white fluffy mould. Under conditions that are favourable to the mould, affected leaves will ultimately shrivel up completely. The stems display large elongated lesions with a brown colour, reaching around the entire stem. The tubers are affected via buds or cracks in the skin; contamination can occur both during growth, harvesting and storage. Damage manifests itself as bluish spots becoming apparent from behind the skin, that turn rusty brown over time. Often, infestation of the tuber leads to dry rot or wet rot (bacterial infection) and the wet rot may then be passed on to unaffected tubers. In this way a limited number of tubers affected by Phytophthora infestans can eventually lead to significant losses during storage.

Photograph: A potato field with advanced Phytophthora infestation.

Phytophthora thrives

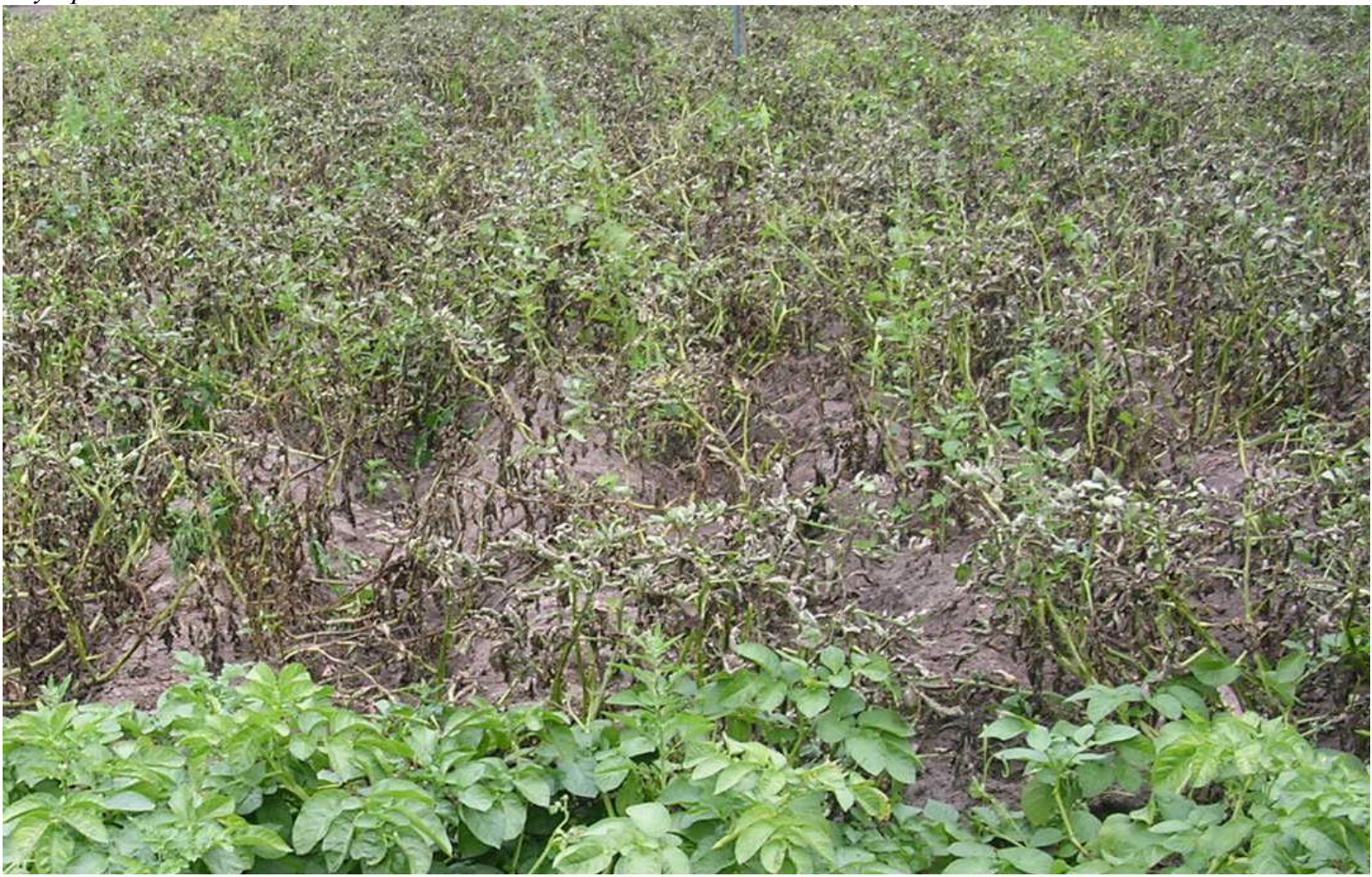

Fig 1: A potato field with advanced Phytophthora infestation

Phytophthora thrives best under high temperatures and under relatively high humidity. Quick penetration of the leave requires a sufficiently long wet leaf period. The disease especially spreads via asexual spores (zoospores) which are formed by the white fluffy mould. The disease is highly contagious. The spores are dispersed via wind or splashing rain drops. 


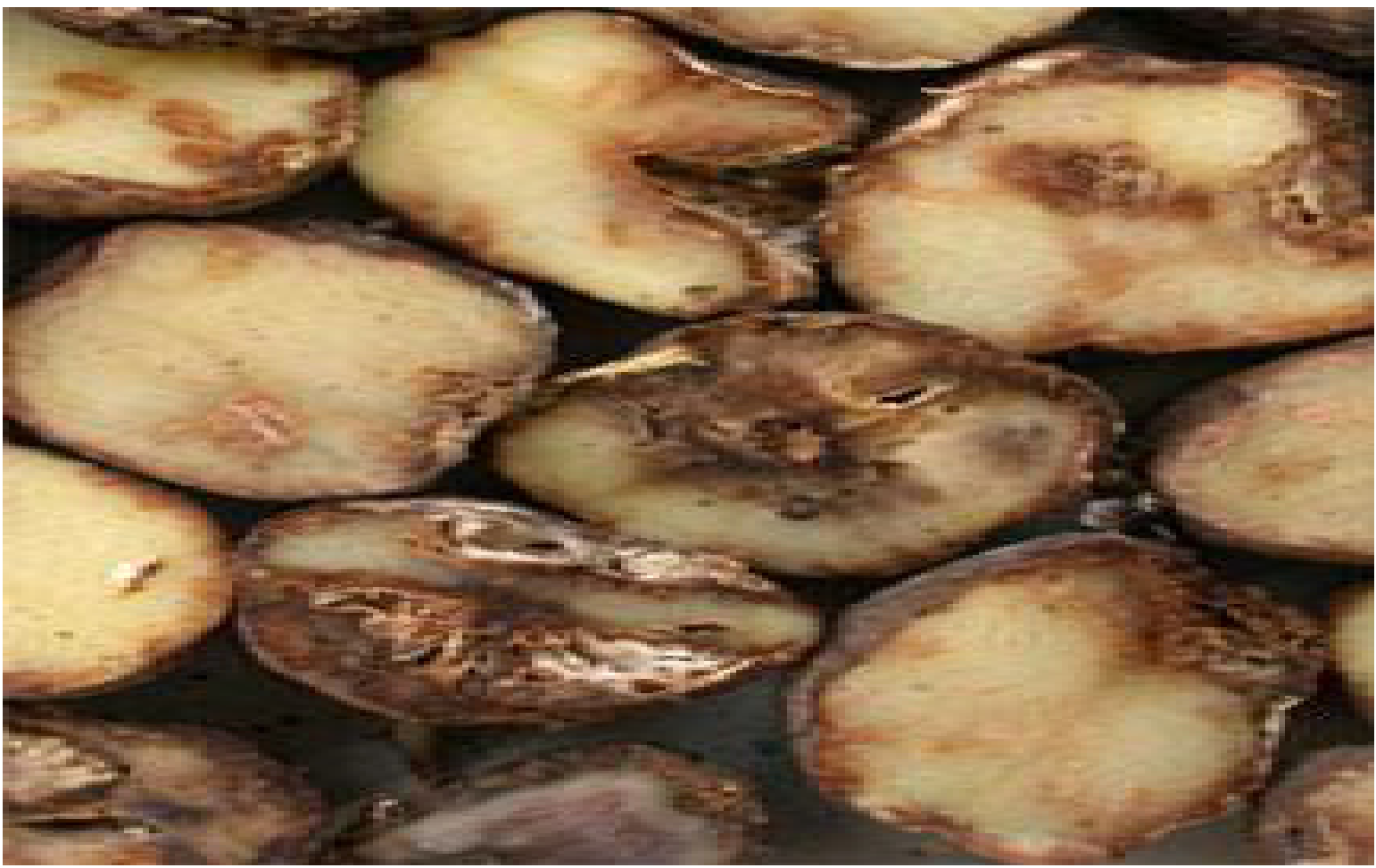

FIG: The result of Phytophthora in affected tubers

Phytophthora infestans survives winter as hyphae in affected tubers. Potato waste heaps are notorious hideouts for the disease. Volunteer plants from tubers left in the field are also an important way in which the disease can survive winter. Since a number of years, however, the disease can also survive via sexual spores (oospores).

Controlling the disease became more difficult over the years, as more aggressive isolates were naturally selected within the Phytophthora infestans population and diversity has increased due to recombination among various mating types previously not occurring within the same area. The increasing aggressiveness is expressed in a shorter penetration time and the disease's life cycle, more generations in a single growth season and a larger temperature tolerance.

\section{2 . Phytophthora infestans causes huge costs}

To prevent the damage require high costs for controlling the disease (costs for purchasing fungicides, plus costs for spray operations) and the costs that result from reductions in harvest and losses during storage. And large quantities of fungicide are used annually for ensuring sufficient control of Phytophthora which have negative impact to the environment.

\subsection{Ways to keep the disease pressure of Phytophthora low}

Important measures of reducing the disease pressure are controlling volunteer plants and destruction of potatoes in waste heaps. In some countries requirements exist to this purpose. Doing this prevents Phytophthora pockets from further spreading the disease, but it does not help in controlling sexual spores (oospores). Crop rotation that is sufficiently slow is effective against spreading the disease via oospores. A second way to reduce disease pressure is by carefully following the instructions of the monitoring services. A last resort is reducing potato cultivation itself - the less potato production, the lower the disease pressure, but of course that is hard to achieve in a country that is famous for its consumption of chips.

\subsubsection{Growing resistant varieties is the best way to reduce disease pressure}

In a country with intensive potato production the best way to reduce disease pressure is growing resistant varieties. The larger the area of cultivated resistant varieties, the lower the disease pressure. In doing this, it is important to insert a sufficient number of different resistance genes. If not, selection pressure will get too high or the limited amount of resistance genes will lead to a quick adaptation of the Phytophthora population.

The area of cultivated resistant varieties probably does not need to be $100 \%$ to sufficiently reduce the disease pressure.

\subsubsection{Acting fast is essential when disease occurs}

Acting fast becomes essential when Phytophthora is found in a field. In practice, the disease is controlled by applying fungicides. Preventive action (treatment before penetration by the mould of the plant takes place) is 
needed to get adequate results. Only a limited number of agents have a sufficiently effective result.

Destruction of potato leafs and stems is another efficient method to prevent the disease from spreading outside the field. However, leaf and stem destruction at a time when the crop is not yet ready for the harvest has significant consequences. Certainly in late maturing varieties strong tuber growth occurs in the last weeks before harvesting. Prematurely destructing the potato leafs and stems would then result in lost yield potential.

\subsubsection{Improved resistance with conventional plant breeding techniques}

It can be done by crossbreed natural resistance genes into commercially useful potato varieties. The resistance genes come from wild, tuber-forming Solanaceae from the Andes.

Crossing in resistance genes to the cultivated potato in a traditional way takes lots of work and patience. This is due to the fact that the potato can not always be crossbred directly with wild relatives in which the resistance genes are present. This necessitates application of a more complicated crossing diagram, using socalled 'bridge cross breeding'. It only allows crossing in one resistance gene at a time, unless you have the luck that the wild variant contains several resistance genes and that at the same time those resistance genes are very close together in the DNA of that wild variant. Furthermore, after crossbreeding with wild variants, many backcrossings are needed to arrive back at the desired cultivated potato properties.

\subsubsection{Phytophthora easily overcomes single resistance genes}

It is known that Phytophthora easily overcomes single resistances. The rich genetic diversity within Phytophthora infestans populations implies that genotypes are already present or emerge (the so-called mutants) and that these can circumvent the mechanism of the single resistance gene that is present. This allows these mutants to have a selective advantage vis-à-vis the wild types and subsequently quickly grow in numbers, dominating the population after a short while. When several resistance genes are present in a potato variety, it is much more difficult for the mould to adjust itself to both resistance genes at the same time. As stated earlier, the varieties Bionica and Toluca contain the single resistance gene Rpi-blb2. This resistance has not been overcome in the field for the moment. The varieties still offer adequate protection. The fact that the varieties are cultivated on a small scale only - mainly by organic and non-commercial vegetable gardeners - means that the selection pressure to overcome the resistance is still limited. However, it is known that isolates exist on which the Rpiblb2 gene has no effect. That is why in The Netherlands the growers of varieties Bionica and Toluca are explicitly asked to keep a close eye on their crop and immediately destruct the leafs and stems if they observe any sign indicating the presence of Phytophthora. If they

\subsubsection{Introducing resistance against Phytophthora infestans by means of genetic manipulation}

Since a number of years resistance genes are also introduced into potatoes by means of genetic modification. This takes place in the U.S. (Cornell University), the U.K. (Sainsbury Laboratory etc.. The advantage of the technology of genetic modification vis-à-vis classical plant breeding is that one can develop resistant varieties much faster and targeted and can also insert several resistance genes at the same time. Furthermore, this insertion via genetic modification can be done without losing properties of the variety.

\subsubsection{Genetic modification for resistance against Phytophthora infestans step-wise} STEP 1: Identifying resistance genes

For making potatoes resistant to Phytophthora, the resistance genes should be obtained first. To do this, wild relatives of our cultivated potato are sown and tested for their resistance. This is initially done with a simple in vitro test. This way, thousands of different wild genotypes have been tested. Also, a so-called DNA fingerprint is made for each of those plants. Next, a leaf test with a number of specific Phytophthora isolates is performed on the plants that demonstrate resistance; this is to understand more about the interaction with the mould. The plants that demonstrate resistance in these tests as well are then subjected to field tests. In this way a smaller number of resistant genotypes remain.

By comparing the DNA of the resistant plants with the plants that are not resistant, the areas in that DNA can be located where the resistance is supposed to lay. Nowadays, the further quest for resistance genes is made easier by the fact that the potato genome at the base pair level is fully sequenced: the DNA order is known every letter of it. Every year, several new resistance genes are discovered. Today, about two dozen different resistance genes are known and available. The isolated genes are also known as cisgenes because they come from varieties that can be crossbred, which can also be used for classical plant breeding.

\section{STEP 2: Modification of potato lines}

Once you have a resistance gene, it can be inserted into the potato. This procedure works as follows: as a first step, the gene is inserted into the DNA of soil bacterium Agrobacterium tumefaciens. It is built into a piece of the bacterium's DNA that is naturally transmitted to plant DNA if the bacterium infects a plant.

Small pieces of potato tissue are brought onto a Petri dish in which a growing medium is present in which the plant can grow. Next, the modified Agrobacterium is added to this potato tissue. The bacterium infects the tissue and transmits the resistance genes to the plant's DNA. Then, a new plant can be generated from the potato tissue. This can be done by adding various plant hormones to the growing medium, inducing the tissue to develop roots and shoots. 
Not all cells are infected by Agrobacterium, so the desired DNA fragment is not present in all plant tissue cells. Therefore, plants should be selected that have actually incorporated the DNA. This can be done in two ways. The first way is to couple another gene to the resistance gene: a so-called selection marker. This can be an antibiotics resistance gene, for example, or a gene providing herbicide tolerance. By then adding the antibiotic or herbicide to the growing medium, only the plants survive that actually incorporated the DNA. This is a simple way of selection.

The selection markers are often obtained from bacteria or from plants with which the potato cannot naturally crossbreed. In other words, these are heterologous. Plants, in which heterologous genes are inserted via genetic modification, are also known as 'transgenic'.

In the second method of selection no selection marker is added to the DNA that is to be transmitted. $\mathrm{Al}$ regenerated plants are allowed to grow up, including the ones that did not incorporate DNA. Next, DNA is obtained from all developed plants. A genetic test based on PCR1 determines whether a particular plant has incorporated the desired DNA. This selection method is a lot more complicated and time-consuming. Plants that have not incorporated the DNA are disposed of.

$1 \mathrm{PCR}=$ Polymerase Chain Reaction

The resistant plants with selection markers, only contain DNA that is inserted via genetic modification from potatoes or plants with which the potato can naturally crossbreed. Such plants are also known as 'cisgenic'. Cisgenic plants to a high degree are comparable with plants which can be obtained via classical plant breeding. The only difference is the technology with which the DNA is introduced.

\section{STEP 3: Testing the plants in a greenhouse}

The first growth stages of the genetically modified potatoes usually take place in a plant nursery room. When the plants have become a bit bigger, they are brought to a greenhouse, where they can continue growth in a pot or in the soil. Growing them in the greenhouse has various purposes. Firstly, it is mainly about establishing whether the desired properties are present. For this, Phytophthora resistance is the dominant important property. Work only continues with plants that demonstrate resistance under a simple leaf test. Also, variety characteristics are checked. Are all relevant properties still there? Variety properties may be lost as a result of any of the next three phenomena: (1) 'insertional mutagenesis', (2) 'pleiotrophy', of (3) 'somaclonal variation'.

Insertion mutagenesis indicates a mistake resulting from insertion of the additional piece of DNA, which ends up in an existing gene, disrupting the function of this particular gene. The potato is tetraploid, which means that it contains 4 copies of each chromosome (4 'homologous chromosomes'). The inserted DNA ends up in one of those four chromosomes, which means that even if the modification leads to a mutation, this will involve only one of the four genes present in the four homologous chromosomes. So the other three genes remain intact. Therefore, any mutation resulting from insertion of DNA will almost never lead to a noticeable change in potato properties.

Pleiotrophy is the phenomenon which involves unexpected and undesirable effects resulting from the fact that the additional piece of DNA ends up in an unexpected place of the plant's DNA. This might for example disrupt the functioning of nearby genes.

Somaclonal variation is the emergence of phenotypic (= observable) deviations from the potato from which it was derived, as a result of growing the plant in vitro and newly regenerating the potato. This latter phenomenon is not specific for the technology of genetic modification and also occurs during regular in vitro reproduction.

Therefore, work in the greenhouse requires strict selection, only selecting those plants that are true-to-type, in other words, which still have all relevant variety properties. The plants displaying resistance AND which are true-to-type are used for further work. With this material, leaf tests are performed with real Phytophthora isolates. And plants that also do well in these tests can in principle qualify for field tests.

\subsubsection{Cultivation of conventional resistant varieties}

Potato varieties differ in their susceptibility for Phytophthora. Varieties such as Bintje and Nicola are highly susceptible to the disease. Early varieties may potentially escape the biggest part of the Phytophthora pressure due to their short growth cycle. Only a small number of varieties such as Bionica, Toluca and Sarpo Mira contain true resistance based on one or several R genes. The resistance genes coming from Solanum demissum that are present in some varieties have often been overcome and therefore cannot be used anymore. Bionica, Toluca and Sarpo Mira are marketed by Dutch firms Meijer and Agrico and Danish firm Danespo, respectively. Bionica and Toluca are only cultivated for the market for fresh potatoes. Bionica and Toluca potatoes are mainly used for organic farming. The potatoes are on the market since a number of years and in 2009 their field resistance still turned out to be good. In Belgium, Bionica is sold to non-commercial vegetable gardeners via AVEVE. In 2010 AVEVE sold seed potatoes to non-commercial growers, translating to over 10 hectares of Bionica cultivation. Of course this is only a drop in the ocean, but non-commercial cultivation is traditionally seen as a potential source of spreading Phytophthora to commercial cultivation, because Phytophthora is hardly controlled in noncommercial cultivation. In this way, a very small number of Bionica can still have an impact on disease pressure. 
Sarpo Mira still is not greatly appreciated by consumers due to its white/yellowish colour on the inside, the flowery cooking result and its not-so-smooth tuber face (faint red skin, irregular shape). The potato however turned out to be well suited for chips and flakes, granulates and mush. McCain exclusively uses Sarpo Mira for their organic chips.

\subsubsection{Sustainable resistance}

Single resistances against Phytophthora are unsustainable. Phytophthora will overcome such a resistance sooner or later, and when that happens, the varieties with such single resistances lose a great deal of their value. Combination of two different resistance genes offers a higher degree of sustainability. This makes it not twice as difficult, but many times as difficult for Phytophthora to overcome the resistance. Triple resistance will provide sustainability that is still many times higher.

The most sustainable resistance is obtained if potato varieties with different multiple resistances are alternated in space and time. Scientists are convinced that in such a scenario - supplemented with some spraying - problems with Phytophthora should be a thing of the past, as Phytophthora would not stand a chance of adapting itself.

\subsubsection{The DuRPh cis genic project}

The DuRPh project started in The Netherlands in 2006. DuRPh stands for ' $\boldsymbol{D} u$ urzame $\boldsymbol{R}$ esistentie tegen Phytophthora' or Sustainable Resistance against Phytophthora. This is a 10-year project financed by the Dutch government which aims at identifying and characterizing resistance genes, creating and testing genetically modified potato lines, and communicate this to a large target audience. The ultimate goal is to present a proof of principle with regard to sustainable resistance against this disease. The project was set up from a genuine sustainability approach and takes into account economic aspects, as well as ecological and social aspects. Wageningen UR took the initiative for the DuRPh project and carries it out.

In the DuRPh project, genetically modified potatoes are developed with a specific underlying idea. Of course the technology of genetic modification is used, and the end result is still subject to GMO legislation, but the plants that are created have the highest possible degree of similarity with plants that can also be obtained via conventional plant breeding. This is called cisgenesis, which implies that:

(1).The resistance genes come from Solanaceae with which the potato can naturally crossbreed.

(2) The resistance genes are not tampered with. They are inserted with their natural regulation signals.

(3) No selection markers will be available in the final lines, only cisgenes. Work with constructs in which a marker is present is used in the first phases of development only, just because this is easier and takes less time, and a quicker insight is obtained in which genes (gene combinations) provide adequate and sustainable resistance.

Since 2006, Wageningen UR performs small-scale field tests with genetically modified Phytophthoraresistant potato lines on various locations in The Netherlands. In these field tests, the various lines that display adequate resistance during greenhouse testing are subjected to a first reality check. In the course of years, various constructs and lines are tested in this way. There are potatoes that include single resistance, double resistance, triple resistance, and lines with (transgenic) and without selection marker (cisgenic

\subsection{Genetically modified Phytophthora-resistant potatoes do not threaten the environment}

Genetically modified plants should not have negative effects on the environment, such as pushing out existing species in nature or disturbing delicate natural balances. Genetically modified Phytophthora-resistant potatoes include resistance genes that naturally occur in wild, tuber-forming Solanaceae. The same or comparable genes are also present in Phytophthora-resistant potatoes that are obtained via conventional plant breeding. Therefore, the genes in the genetically modified potato lines are just as safe to the environment as the same genes in the varieties obtained via conventional plant breeding and those in wild relatives. Is that the end of the story? Not quite. It cannot be completely ruled out that genetic modification leads to unexpected phenomena having an effect on the survival of the potato plant in nature, or that other unexpected changes in plant properties emerge. For this reason, strict selection takes place during the quest for suitable genetically modified lines, in order to only select those lines that kept the right variant properties. In field tests, the phenotype and behaviour of the plants also undergo exhaustive scrutiny in order to locate any deviations, and to look whether deviations have undesirable consequences. Plants with undesirable properties will not be marketed.

In Europe, the potato is absent as a wild plant. Furthermore, the potato has no relatives with which it could crossbreed. Black nightshade is phylogenetically closest to the potato, but cannot be interbred with it. Furthermore, the cultivated potato is a highly domesticated plant that will not suddenly colonise nature. The plant lacks the power to successfully compete with wild plants. In practice, this means that neither the genetically modified potatoes, nor the genetically modified property itself will be able to colonise our environment. The potato will only be able to appear as volunteer plants in fields where it has previously grown, and in soil coming from such fields. These cases will, however, always be a temporary affair. the genetically modified potato lines are just as safe to the environment as the same genes in the varieties obtained via conventional plant breeding and those in wild relatives. Is that the end of the story? Not quite. It cannot be 
completely ruled out that genetic modification leads to unexpected phenomena having an effect on the survival of the potato plant in nature, or that other unexpected changes in plant properties emerge. For this reason, strict selection takes place during the quest for suitable genetically modified lines, in order to only select those lines that kept the right variant properties. In field tests, the phenotype and behaviour of the plants also undergo exhaustive scrutiny in order to locate any deviations, and to look whether deviations have undesirable consequences. Plants with undesirable properties will not be marketed.

In Europe, the potato is absent as a wild plant. Furthermore, the potato has no relatives with which it could crossbreed. Black nightshade is phylogenetically closest to the potato, but cannot be interbred with it. Furthermore, the cultivated potato is a highly domesticated plant that will not suddenly colonise nature. The plant lacks the power to successfully compete with wild plants. In practice, this means that neither the genetically modified potatoes, nor the genetically modified property itself will be able to colonise our environment. The potato will only be able to appear as volunteer plants in fields where it has previously grown, and in soil coming from such fields. These cases will, however, always be a temporary affair.

\subsection{Food safety of genetically modified Phytophthora-resistant potatoes}

Genetically modified Phytophthora-resistant potatoes contain the same or comparable resistance genes as traditional Phytophthora-resistant potatoes do. To provide a practical example: BASF's genetically modified Phytophthora potato and the potato varieties Bionica and Toluca that have been obtained via conventional plant breeding, all contain the same Rpi-blb2 gene. For the genetically modified potatoes, the safety of the gene product that is synthesized by the resistance gene should be demonstrated. It should be demonstrated that the product is not toxic and does not cause allergic reactions. This is not a requirement in varieties like Bionica or Toluca which are the result of conventional plant breeding. These varieties can be marketed without further food safety tests. And all this despite the fact that the Rpi-blb2 gene in these plants is surrounded by hundreds of genes originating from the wild variety.

As indicated earlier, it cannot be ruled out that genetic modification can lead to unexpected side effects. Insertional mutagenesis, pleiotrophy and somaclonal variation were mentioned as possible causes of such side effects. For this reason, a genetically modified Phytophthora-resistant potato should undergo additional tests. The composition of the potato should be compared with that of conventional potatoes, for example. For this purpose the quantity of the 21

main components of the potato is measured and compared. The OECD determined which substances should be included in the analysis. The potato naturally contains toxic glycoalkaloids. Their presence is the reason why one should peel potatoes before they can be safely eaten. Besides, the potato contains substances blocking our digestive enzymes. Eating raw potatoes leads to stomach-ache, so to prevent this we cook them before consumption. In the required analysis, the quantity of these kinds of substances is closely watched, because everything should be done to prevent marketing potatoes that carry too many hazardous substances. By the way, the amount of glycoalkaloids is checked for every new variety before market entry, whether it is genetically modified or not. In The Netherlands and Sweden an upper safety limit of a total glycoalkaloid level of 200 $\mathrm{mg} / \mathrm{kg}$ applies. Other EU countries have not specified glycoalkaloid limits.

If analysis of the composition of the genetically modified potato shows an unexpected deviation in the level of certain substances, additional tests should be performed. Usually this takes the form of a 90-day food test on rats.

\subsection{Genetically modified Phytophthora-resistant potatoes not on the market for now}

BASF's genetically modified Phytophthora-resistant Fortuna potato reached an advanced stage, but is still not on the market. BASF should first apply for market admission with the European Commission to achieve this. And once that file has been submitted, it will take some years more before the full procedure is completed. It is hard to look into the future, but it is expected to last until 2013 before a genetically modified Phytophthora-resistant potato is available on the market. All other lines that are being developed are less far advanced than the Fortuna potato and their market introduction will take a lot more time.

\section{SUMMARY AND CONCLUSION}

Agriculture is a back bone to bring over all development in the world particularly in developing countries. Now a day's population is ever-increasing but the land is as it is. Therefore increasing production and productivity of the crop is paramount important to assure food and nutrition security for all population and supply inputs to the factories. To realize these tuber crop, potato is the one that is produced in large amount in the world. To improve production and productivity using a combination techniques indispensable. To mention some of the improvement develop resistance varieties to disease and pest of the potato using breeding and gene modification systems. Late blight is a serious disease that is caused by Phytophthora which is a very devastated to potato. Hence developing resistant potato varieties is essential to reduce yield loss of potato by light blight. 


\section{REFERENCES}

Beyer, P., et al. Golden rice: Introducing the $\beta$-carotene biosynthesis pathway into rice endosperm by genetic engineering to defeat vitamin A deficiency. Journal of Nutrition 132, 506S-510S (2002)

Demont, M., et al. GM crops in Europe: How much value and for whom? EuroChoices 6, 46-53 (2007)

Devos, Y., et al. Ethics in the societal debate on genetically modified organisms: A (re)quest for sense and sensibility. Journal of Agricultural and Environmental Ethics 21, 29-61 (2007) doi:10.1007/s10806-0079057-6

Guerrero-Andrade, O., et al. Expression of the Newcastle disease virus fusion protein in transgenic maize and immunological studies. Transgenic Research 15, 455-463(2006) doi:10.1007/s11248-006-0017-0

Hoban, T. Public attitudes towards agricultural biotechnology. ESA working papers nos. 4-9. Agricultural and Development Economics Division, Food and Agricultural Organization of the United Nations (2004)

Jesse, H., \& Obrycki, J. Field deposition of Bt transgenic corn pollen: Lethal effects on the monarch butterfly. Oecologia 125, 241-248 (2000)

Ma, J., et al. The production of recombinant pharmaceutical proteins in plants. Nature Reviews Genetics 4, 794805 (2003) doi:10.1038/nrg1177

Muir, W., \& Howard, R. Possible ecological risks of transgenic organism release when transgenes affect mating success: Sexual selection and the Trojan gene hypothesis. Proceedings of the National Academy of Sciences 96, 13853-13856 (1999)

Sears, M., et al. Impact of Bt corn on monarch butterfly populations: A risk assessment. Proceedings of the National Academy of Sciences 98, 11937-11942 (2001)

Takeda, S., \& Matsuoka, M. Genetic approaches to crop improvement: Responding to environmental and population changes. Nature Reviews Genetics 9, 444-457 (2008) doi:10.1038/nrg2342 . 